\title{
Effect of temperature on the specific energy of $\beta / \alpha$ interfaces in Ti-6Al-4V alloy
}

\author{
M.A. Murzinova ${ }^{\dagger}$ \\ †mma@imsp.ru
}

Institute for Metals Superplasticity Problems RAS, 39 Khalturin St., 450001 Ufa, Russia

\begin{abstract}
Most of titanium alloys contain two phases at temperatures of processing and exploitation: $\alpha$-phase with HCP lattice and $\beta$-phase with BCC lattice. Stability of two-phase structure and thermodynamics of $\beta / \alpha$ transformation, which occurs in a wide range of temperatures, considerably depend on the specific energy of interphase boundaries, and, consequently, on their structure. Basing on the literature data, four schemes of "planar" semicoherent interfaces, in which mismatches in interatomic distances are accommodated by two or three sets of edge misfit dislocations, were plotted. Such types of conjugation are observed on side and end interfaces of lamellas in titanium alloys. The procedure developed by J. H. van der Merwe and G. J. Shiflet was used for estimation of specific energy of interphase boundaries in Ti-6Al-4V alloy. Effect of temperature and content of alloying elements on lattice parameters and elastic properties of the $\alpha$ - and $\beta$-phases in Ti- $6 \mathrm{Al}-4 \mathrm{~V}$ alloy was taken into account for calculations. This approach allowed one to estimate the change in the energy of interphase boundaries in the alloy in temperature interval $600-975^{\circ} \mathrm{C}$, where diffusion $\beta / \alpha$ transformation and enrichment of $\beta$-phase by vanadium are observed. It was shown that the energy of interphase boundaries in Ti-6Al-4V alloy decreased by $1.3-1.5$ times with increasing temperature from 600 to $975^{\circ} \mathrm{C}$ at all examined types of conjugation. Depending on the structure the energy of interphase boundaries can vary from 0.201 to $0.337 \mathrm{~J} / \mathrm{m}^{2}$ at $975^{\circ} \mathrm{C}$ and from 0.298 to $0.429 \mathrm{~J} / \mathrm{m}^{2}$ at $600^{\circ} \mathrm{C}$.
\end{abstract}

Keywords: energy of interphase boundaries, titanium alloys.

\section{Влияние температуры на удельную энергию межфазных $\beta / \alpha$ границ в сплаве Ti-6Al-4V}

\author{
Мурзинова М.А. ${ }^{\dagger}$ \\ †mma@imsp.ru
}

Институт проблем сверхпластичности РАН, ул. Халтурина 39, 450001, Уфа, Россия

Во многих титановых сплавах при температурах обработки и эксплуатации присутствуют две фазы: $\alpha$-фаза с ГПу решеткой и $\beta$-фаза с ОЦК решеткой. Стабильность двухфазной структуры и термодинамика фазового $\beta / \alpha$ превращения, которое протекает в широком интервале температур, во многом зависят от удельной энергии межфазных границ, и, следовательно, их строения. На основе обобщения литературных данных об атомном и дислокационном строении границ между фазами с ОЦК и ГПУ решетками предложены четыре схемы строения «плоских» полукогерентных межфазных границ, на которых несоответствия межатомных расстояний компенсируются двумя или тремя рядами краевых дислокаций несоответствия. Границы сопряжения такого типа наблюдаются на боковых и торцевых гранях пластин в титановых сплавах. По методике, разработанной ван дер Мерве и Шифлитом, выполнена оценка удельной энергии межфазных границ разного типа в сплаве Ti-6Al-4V. Расчеты проводились с учетом литературных данных о влиянии содержания легирующих элементов и температуры на параметры решеток и упругие свойства фаз сплава Ti-6Al-4V. Это позволило оценить изменение энергии межфазных границ в сплаве Ti-6Al-4V в интервале температур $600-975^{\circ} \mathrm{C}$, при которых наблюдается развитие диффузионного $\beta / \alpha$ превращения и интенсивное обогащение $\beta$-фазы ванадием. Показано, что при всех вариантах сопряжения энергия межфазных границ в сплаве Ti-6Al-4V монотонно уменьшается в 1,3 - 1,5 раза с повышением температуры от 600 до $975^{\circ} \mathrm{C}$. При постоянной тем-

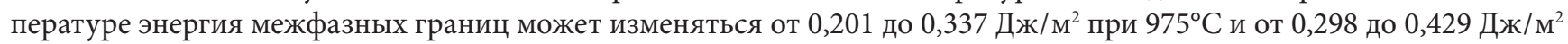
при $600^{\circ} \mathrm{C}$ в зависимости от строения фасеток.

Ключевые слова: энергия межфазных границ, титановые сплавы. 


\section{1. Введение}

Одной из важных характеристик сплавов, претерпевающих фазовые превращения в твердом состоянии, является удельная энергия межфазных границ. От ее величины зависят размер критического зародыша, кинетика фазового превращения, морфология кристаллов образующейся фазы и др. [1-4]. Чем ниже удельная энергия межфазной границы, тем ниже скорость диффузии по межфазным границам, меньше скорость их миграции, тем стабильнее частицы второй фазы [1-6]. Величина энергии межфазных границ также является одним из основных параметров, которые используются при моделировании структурных изменений и свойств сплавов под влиянием внешних воздействий [2,5-9]. Поскольку прямых экспериментальных методов измерения энергии межфазных границ нет, за величину энергии межфазных границ часто принимают долю энергии высокоугловых границ зерен (High angle boundaries $-E_{\text {нАв}}$ ) одной из фаз. Считают, что энергия некогерентных межфазных границ составляет $(0.7-1) E_{\text {НАВ }}$, а полукогерентных $-(0.4-0.5) E_{\mathrm{HAB}}[1,2,9]$. В то же время известно, что энергия межфазных границ определяется их строением, и строение разных граней сопрягающихся фаз существенно различается, особенно в тех случаях, когда между фазами существует определенное кристаллографическое ориентационное соответствие $[1-4]$. Например, для $(\alpha+\beta)$-сплавов титана c пластинчатой микроструктурой показано, что между высокотемпературной $\beta$-фазой с объемно-центрированной кубической решеткой (ОЦК) и низкотемпературной $\alpha$-фазой с гексагональной плотноупакованной (ГПУ) решеткой наблюдается ориентационное соотношение (ОС) Бюргерса $\left((0001)_{\alpha}\left\|\{110\}_{\beta},\langle 11 \overline{2} 0\rangle_{\alpha}\right\|\langle 1 \overline{1} 1\rangle_{\beta}\right.$, иногда слабое отклонение от него), и на разных гранях сопрягающихся пластин присутствуют дислокации несоответствия [10-19]. Один из возможных вариантов пространственного расположения элементарных ГПУ и ОЦК ячеек, обеспечивающих выполнение ОС Бюргерса, показан на рис. 1a. На рис. $1 \mathrm{~b}$ представлена трехмерная модель сопряжения $\beta$ и $\alpha$ фаз, построенная на основе обобщения результатов моделирования и экс- периментальных исследований [10-21]. Необходимо отметить, что единого мнения о плоскостях (фасетках) сопряжения разных граней $\alpha$ и $\beta$-пластин и их дислокационном строении нет.

Однако в большинстве случаев на широкой межфазной поверхности (Broad Face) наблюдается один ряд дислокаций несоответствия, вектор Бюргерса которых содержит "c"-компоненту (здесь и далее обозначение дислокаций приводится для $\alpha$-фазы титана или указываются кристаллографические индексы направлений обеих решеток). На боковой поверхности (Side Face) сопряжения присутствуют два ряда дислокаций несоответствия " $a$ "-типа, линии которых расположены, как правило, в плотноупакованных плоскостях $(0001)_{\alpha} \|\{110\}_{\beta} \alpha$ - или $\beta$-фазы, а на торцевой поверхности (End Facet) пластин наблюдали два или три ряда дислокаций несоответствия “ $a$ ", “c” или " $a+c$ "-типа [19] (рис. 1b). Результаты моделирования [11] и высокоразрешающей электронной микроскопии показали $[12,13,20,21]$, что кроме дислокаций несоответствия на широкой поверхности сопряжения $\alpha$ и $\beta$ пластин формируются структурные ступеньки. Присутствие структурных ступенек, террасы которых образованы плоскостями $(01 \overline{1} 0)_{\alpha} \|(1 \overline{1} 2)_{\beta}$, хорошо объясняет выполнение ОС Бюргерса при больших индексах Миллера «макроскопической плоскости» габитуса (рис. 1b). Структурные ступеньки компенсируют несоответствие межатомных расстояний в направлении “ $a$ " $-[\overline{2} 110]_{\alpha} \|[\overline{1} 11]_{\beta}$, а межфазные дислокации с вектором Бюргерса $[0001]_{\alpha} \|[110]_{\beta}-$ в направлении " $c$ ". Модель ступенчатой межфазной границы в сплавах титана, предложенная в [11], и алгоритм расчета энергии ступенчатой границы, разработанный в $[22,23]$, были использованы для оценки изменяя удельной энергии широкой межфазной границы в сплаве ВТ6 в зависимости от температуры обработки в [24].

В данной работе выполнялась оценка изменения удельной энергии боковой и торцевой межфазных $\beta / \alpha$ границ в титановом сплаве Ti-6Al-4V в интервале температур $600-975^{\circ} \mathrm{C}$, при которых наблюдается развитие диффузионного $\beta / \alpha$ превращения и интенсивное обогащение $\beta$-фазы ванадием [25].
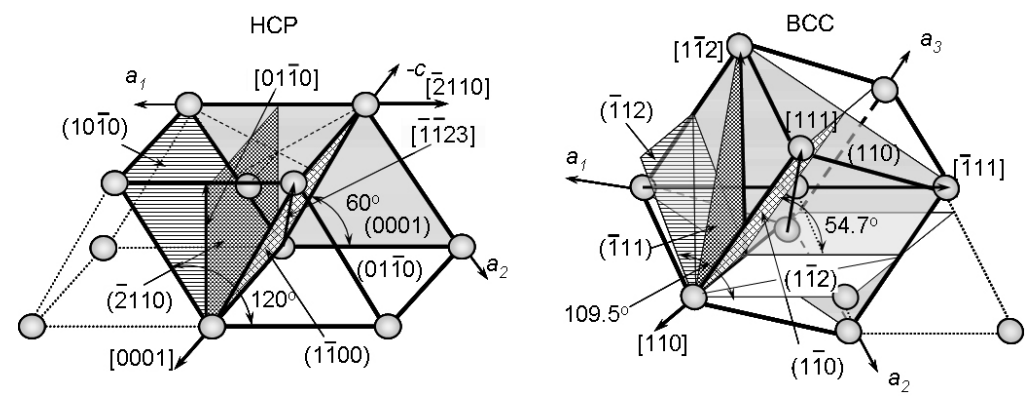

a

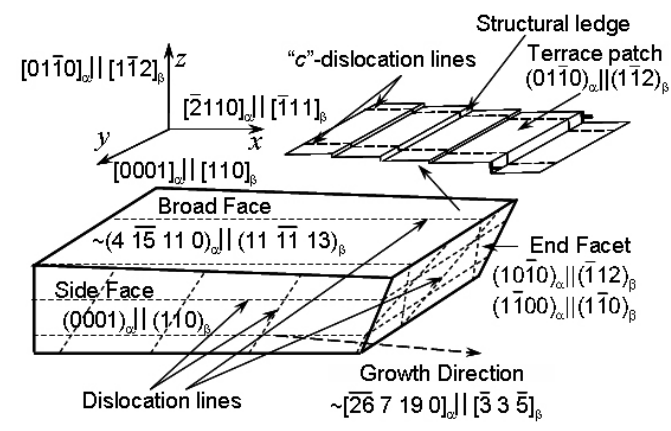

$\mathrm{b}$

Pис. 1. (Color online) (а) Пространственное расположение ОЦК и ГПУ решеток, обеспечивающее выполнение ОС Бюргерса; (b) Трехмерная схема строения межфазных $\beta / \alpha$ границ в сплавах титана (по данным [10-21]).

Fig. 1. (Color online) (a) Spatial arrangement of a Burgers-relate BCC and HCP lattices. (b) Schematic of 3D structure of $\beta / \alpha$ interfaces in titanium alloys (according to [10-21]). 


\section{2. Оценка энергии $\beta / \alpha$ границ в сплаве Ti-6Al-4V}

1. Схематическое и кристаллографическое строение

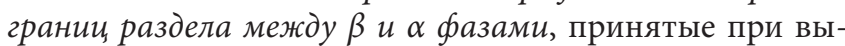
полнении оценок их энергии, представлены на рис. 2. Как и в работе [24], эти схемы предполагают выполнение ОС Бюргерса, присутствие на широкой границе раздела фаз структурных ступенек и одного ряда краевых “c”-дислокаций несоответствия, а также параллельность плотноупакованных плоскостей ОЦК и ГПУ решеток на боковой поверхности пластин. Несоответствие межатомных расстояний на боковой границе компенсируется двумя рядами краевых “ $a$ ”-дислокаций несоответствия с векторами Бюргерса $\mathbf{b}_{1}=1 / 3[\overline{2} 110]_{\alpha} \| 1 / 2[\overline{1} 11]_{\beta}$ и $\mathbf{b}_{2}=1 / 3[\overline{1} \overline{1} 20]_{\alpha} \|[001]_{\beta}$ (рис. 2a). Заметим, что при строгом выполнении условия $[\overline{2} 110]_{\alpha} \|[\overline{1} 11]_{\beta}$ угол между направлениями $[\overline{1} 120]_{\alpha}$ и $[001]_{\beta}$ составляет примерно $5,3^{\circ}$. Тем не менее, возможность присутствия межфазных дислокаций несоответствия с такими векторами Бюргерса показана в $[11,19,20]$.

Строение торцевой $\beta / \alpha$ границы вызывает наибольшие дискуссии $[11,12,19]$. Вероятно, это связано с тем, что торцевая поверхность раздела может состоять из фасеток, образованных разными парами плоскостей. Например, плоскостями $(\overline{2} 110)_{\alpha} \|(\overline{1} 11)_{\beta}$, которые перпендикулярны плоскостям террасы и боковой грани $[11,24]$, а также парами плоскостей $(10 \overline{1} 0)_{\alpha}$ и $(\overline{1} 12)_{\beta}$, или $(1 \overline{1} 00)_{\alpha}$ и $(1 \overline{1} 0)_{\beta}$ (рис. 1a). Все эти плоскости содержат направление $[0001]_{\alpha} \|[110]_{\beta}$, в котором несоответствие межатомных расстояний может быть скомпенсировано межфазными дислокациями “c”-типа. Кроме того, на торцевой грани наблюдали дислокации несоответствия “ $a$ ” $[11,12]$ и “ $a+c$ "-типа [19].

Присутствие фасеток, образованных плоскостями $(\overline{2} 110)_{\alpha} \|(\overline{1} 11)_{\beta}$ и имеющих дислокационное строение, маловероятно, т. к. в интервале температур $600-975^{\circ} \mathrm{C}$ несоответствие межатомных расстояний в направлении $[01 \overline{1} 0]_{\alpha} \|[1 \overline{1} 2]_{\beta}$, составляет $43,3-44,5 \%$, и расстояния между дислокациями несоответствия в этом случае соизмеримы с межатомными. Образование фасеток сопряжения по плоскостям $(10 \overline{1} 0)_{\alpha}$ и $(\overline{1} 12)_{\beta}$, с дислокациями несоответствия “ $a$ ”-типа (вектор Бюргерса $\mathbf{b}_{1}$ ) и “с"-типа (вектор Бюргерса $\mathbf{b}_{3}=[0001]_{\alpha} \|[110]_{\beta}$ ) (рис. 2b), предполагает взаимный разворот этих плоскостей на угол до $10,5^{\circ}$ (рис. 1a). К такому развороту может привести, например, формирование границ наклона в сопрягающихся фазах. Образование фасеток сопряжения по плоскостям $(1 \overline{1} 00)_{\alpha}$ и $(1 \overline{1} 0)_{\beta}$ тоже предполагает их разворот, но на меньший угол - до 5,3․ В этих плоскостях несоответствия межатомных расстояний могут быть скомпенсированы двумя или тремя рядами краевых дислокаций несоответствия с векторами Бюргера $\mathbf{b}_{2}$ и $\mathbf{b}_{\mathbf{3}}$ или $\mathbf{b}_{2}, \mathbf{b}_{3}$ и $\mathbf{b}_{4}=1 / 6[\overline{1} 123]_{\alpha} \| 1 / 2[111]_{\beta}$, соответственно (рис. 2c). В данной работе была выполнена оценка удельной энергии межфазных границ для всех случаев сопряжения, показанных на рис. 2.

2. Энергию межфазных грании, оценивали по алгоритму, описанному в [26]. Расчетные формулы подробно описаны в первоисточниках [26,3] и в книгах по физике металлов [например, 1,2] (эти выражения весьма громоздкие, и здесь не приводятся). При оценке энергии плоской границы с межфазными дислокациями несоответствия учитывается: энергия упругих смещений атомов в плоскости сопряжения, которые происходят в направлениях векторов Бюргерса дислокаций несоответствия, энергия упругих смещений атомов в направлении, нормальном к плоскости границы, и релаксация упругих напряжений, обусловленная дислокациями несоответствия. Показано [26], что для оценки энергии межфазной границы достаточно знать параметры решеток, модули сдвига и коэффициенты Пуассона сопрягающихся фаз. Зная параметры решеток, по формулам кристаллографии [1] легко определить межатомные и межплоскостные расстояния для заданных кристаллографических плоскостей сопряжения.

Химический состав и параметры решеток $\alpha$ - и $\beta$-фазы в сплаве Ti-6Al-4V при температурах от 600 до $975^{\circ} \mathrm{C}$ экспериментально измерены в [25]. Модули сдвига и коэффициенты Пуассона фаз с заданным химическим составом при каждой соответствующей температуре определены в [24]. Эти данные позволили оценить величину удельной энергии межфазных границ в соответствии с их строением (рис. 2).

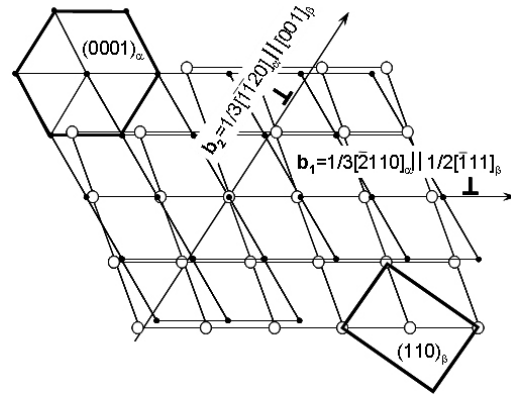

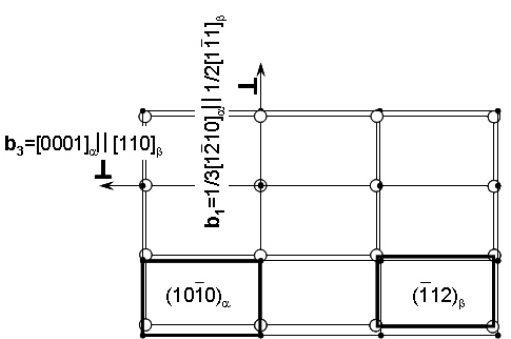

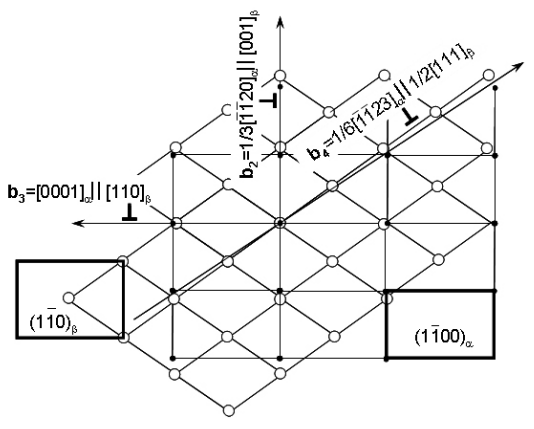

C

Рис. 2. Схемы расположения атомов в плоскостях сопряжения ОЦК/ГПУ решеток и векторы Бюргерса межфазных дислокаций несоответствия: (a) на боковой поверхности; (b) на торцевой фасетке, образованной плоскостями (1010) и (1 12) $)_{\beta}$ (c) на торцевой фасетке, образованной плоскостями $(1 \overline{1} 00)_{\alpha}$ и $(1 \overline{1} 0)_{\beta}$.

Fig. 2. Atomic patterns in conjugated planes of the BCC/HCP lattices and Burgers vectors of misfit dislocations on: (a) side face; (b) end facet formed by $(10 \overline{1} 0)_{\alpha}$ and $(\overline{1} 12)_{\beta}$ planes; (c) end facet formed by $(1 \overline{1} 00)_{\alpha}$ and $(1 \overline{1} 0)_{\beta}$ planes. 
Результаты выполненной оценки представлены на рис. 3. С повышением температуры от 600 до $975^{\circ} \mathrm{C}$ удельная энергия всех межфазных $\beta / \alpha$ границ снижается. Наименьшую удельную энергию имеет ступенчатая широкая межфазная граница, наибольшую - торцевая граница, образованная парой плоскостей $(1 \overline{1} 00)_{\alpha} \|(1 \overline{1} 0)_{\beta}$, как с тремя, так и с двумя рядами дислокаций несоответствия. Эти результаты соответствуют трехмерной схеме (рис. 1b), т. к. границы с большей энергией обычно имеют меньшую площадь и большую диффузионную подвижность и наоборот [1-4]. Величины удельной энергии боковой границы, где сопрягаются плотноупакованные плоскости $(0001)_{\alpha} \|(110)_{\beta}$, и торцевой, образованной плоскостями $(10 \overline{1} 0)_{\alpha}$ и $(\overline{1} 12)_{\beta}$, близки. Однако здесь уместно напомнить, что для обеспечения параллельности плоскостей $(10 \overline{10})_{\alpha}$ и $(\overline{1} 12)_{\beta}$ необходим их разворот на угол около $10,5^{\circ}$. Во всем интервале температур развития $\beta / \alpha$ превращения коэффициент анизотропии удельной межфазной поверхностной энергии $\left(K=E_{\max } / E_{\min }\right)$ близок к 2. Полученные значения энергии удовлетворительно согласуются с данными, приводимыми в литературе [9], где энергия межфазных границ оценивалась как доля энергии высокоугловых границ зерен.

Использованный в данной работе подход к оценке удельной энергии $\beta / \alpha$ границ в сплавах титана имеет ряд ограничений. В частности, этот подход: а) не учитывает анизотропию упругих свойств исходной и образующейся фазы; б) не учитывает вклад винтовых компонент межфазных дислокаций несоответствия, которые могут присутствовать на межфазных границах [3,11,14-19]; в) учитывает только структурную составляющую межфазной поверхностной энергии, пренебрегая химической, вклад которой может быть существенным при низких температурах [2]; г) не учитывает вклад атомов «второго слоя» в решетках сопрягающихся фаз в компенсацию несоответствия на дислокационных межфазных границах.

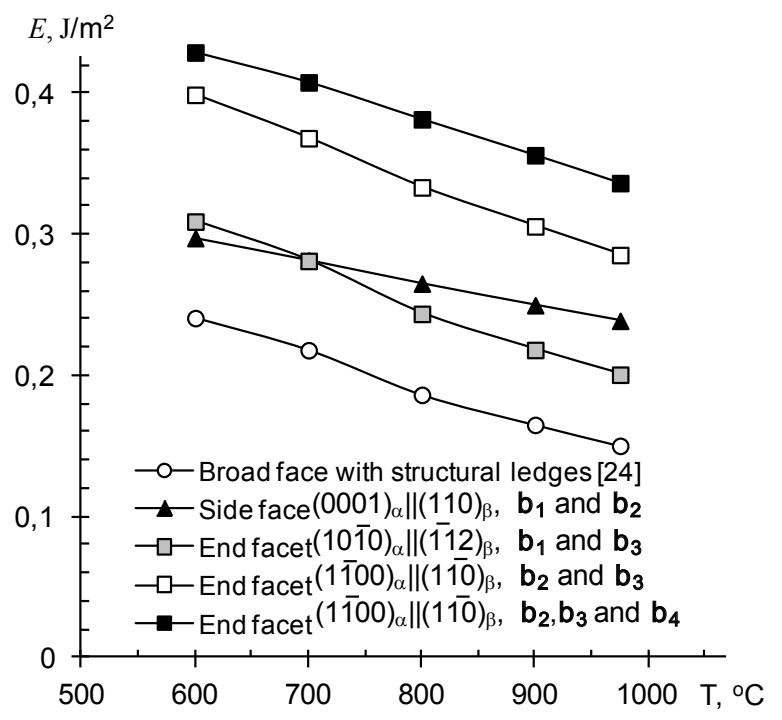

Рис. 3. Влияние температуры на удельную энергию межфазных $\beta / \alpha$ границ в сплаве Ti-6Al-4V.

Fig. 3. Effect of temperature on specific energy of the $\beta / \alpha$ interfaces in Ti-6Al-4V alloy.

\section{3. Заключение}

На основе обобщения литературных данных о строении боковой и торцевой границ между $\alpha$ и $\beta$ пластинами в сплавах титана предложены схемы строения межфазных границ, на которых присутствуют два или три ряда краевых дислокаций несоответствия. По методике [26] выполнена оценка энергии этих межфазных границ в сплаве Ti-6Al-4V в интервале температур $600-975^{\circ} \mathrm{C}$. Показано, что повышение температуры приводит к уменьшению удельной поверхностной энергии всех рассмотренных межфазных границ в $1,3-1,5$ раза и не оказывает значимого влияния на ее анизотропию. Границы, образованные плоскостями $(0001)_{\alpha} \|(110)_{\beta}$ и $(10 \overline{1} 0)_{\alpha} \|(\overline{1} 12)_{\beta}$ с двумя рядами дислокаций несоответствия, имеют близкую удельную энергию, которая при $600^{\circ} \mathrm{C}$ составляет около 0,3 Дж/ м $^{2}$. Большую энергию имеет граница,образованнаяплоскостями $(1 \overline{1} 00)_{\alpha} \|(1 \overline{1} 0)_{\beta}$ : при $600^{\circ} \mathrm{C}$ ее энергия составляет 0,4 Дж/ $\mathrm{M}^{2}$ в присутствии двух рядов дислокаций несоответствия, и 0,43 Дж/ $\mathrm{m}^{2}-$ в присутствии трех рядов дислокаций.

\section{Литература/References}

1. A. Kelly, G.W. Groves. Crystallography and crystal defects. Longman, London, (1970).

2. D.E. Laughlin, K. Hono eds. Physical Metallurgy: 3-Volume Set, 5th edition, Newnes, (2014), p. 2960.

3. G. J. Shiflet, Mater. Sci. Eng. 81, 61 (1986).

4. J.P. Hirth, G. Spannos, M.G. Hall, H.I. Aaronson. Mechanisms for the development of tent-shaped and invariant-plane-strain-type surface reliefs for plates formed during diffusional phase transformations.. Acta Materialia 46 No.3, (1998) 857 - 868.

5. S.Z. Bokshtein (Ed.) Diffusion Processes, Structure, and Properties of Metals. Springer; Softcover reprint of the original 1st ed. 1965 edition (December 16, 2013), p.135.

6. S.L. Semiatin, D.U. Furrer, in ASM Handbook, Fundamentals of Modeling for Metals Processing, ed. by S. L. Semiatin, D. U. Furrer, Materials Park, OH: ASM International, (2009), Vol. 22, p. 536.

7. W. Sha, S. Malinov. Titanium alloys: modelling of microstructure, properties and applications. First published 2009, Woodhead Publishing Limited and CRC Press LLC, (2009), p. 598.

8. J. Da Costa Teixeira, B. Appolaire, E. Aeby-Gautier, S. Denis, L. He 'richer, Computational Materials Science 42, 266 (2008).

9. R. Shi, N. Ma, Y. Wang, Acta Materialia 60, 4172 (2012).

10. T. Furuhara, H. J. Lee, E.S. K. Menon, H.I. Aaronson, Metall. Trans. A 21, 1627 (1990).

11. T. Furuhara, H. I. Aaronson, Acta Metall. Mater. 39, 2857 (1991).

12. T. Furuhara, J.M. Howe, H.I. Aaronson, Acta Metall. Mater. 39, 2873 (1991).

13. T. Furuhara, T. Ogawa, T. Maki, Phil. Mag. Lett. 72, 175 (1995)

14. E. S. K. Menon, H.I. Aaronson, Acta Metall. Mater. 39, 1975 (1986).

15. N. Miyano, A. Takahashi, J. Miyazaki, K. Ameyama, 
Mater. Trans. 49, 471 (2008).

16. N. Miyano, K. Ameyama, G. C. Weatherly, Mater. Trans. 43, 1547 (2002).

17. S. Suri, G.B. Viswanathan, T. Neeraj, D.-H. Houi, M. J. Mills, Acta mater. 47, 1019 (1999).

18. F. Ye, W.-Z. Zhang," D. Qiu, Acta Materialia 52, 2449 (2004).

19. F. Ye, W.-Z. Zhang, Acta Materialia 54, 871 (2006).

20. R. C. Pond, S. Celotto, J.P. Hirth, Acta Mater. 51, 5385 (2003).

21. S. Nag, R. Banerjee, R. Srinivasan, J. Y. Hwang, M. Harper,
H. L. Fraser, Acta Mater. 57, 2136 (2009).

22. J. H. van der Merwe, G. J. Shiflet, P. M. Stoop, Metallurgical Transactions A 22, 1165 (1991).

23. J. H. van der Merwe, G. J. Shiflet, Acta Metal. Mater. 42, 1173 (1994).

24. M.A. Murzinova, S.V. Zherebtsov, G.A. Salishchev, Journal of Experimental and Theoretical Physics 122, 705 (2016).

25. J. W. Elmer, T. A. Palmer, S. S. Babub, E. D. Specht, Mater. Sci. Eng. A 391, 104 (2005).

26. J. H. van der Merwe, Proc. Phys. Soc. A 63, 616 (1950). 